\title{
The E-peak distribution of the GRBs detected by HETE FREGATE instrument
}

\author{
C. Barraud*, J. L. Atteia*, J. F. Olive* and J. P. Dezalay* \\ ${ }^{*}$ Centre d'Etude Spatiale des Rayonnements-CNRS/UPS- 9 Av du Colonel Roche, \\ BP 4346-31 028 Toulouse Cedex 4,FRANCE
}

\begin{abstract}
The FREGATE gamma ray detector of HETE-2 is sensitive to photons between 6 and $400 \mathrm{keV}$. This sensitivity range, extended towards low energies, allows us to explore the emission of GRBs in hard X-rays. We fit the spectra of 23 GRBs with Band's spectral function in order to derive the distribution of their peak energies (E-peak). This distribution is then compared with the E-peak distributions measured by BATSE and GINGA.
\end{abstract}

\section{INTRODUCTION}

We present here a preliminary analysis of the spectral distribution of FREGATE's GRBs. FREGATE is the gamma-ray detector of HETE-2 (see [1] for a description of FREGATE). During its first year of operation (HETE has been launched on october 9th 2000), FREGATE has detected 37 GRB candidates: for 13 of them, a position has been determined, 13 have no localization but are in the field of view and the remaining 11 are out of the field of view.

In this preliminary study, we focus on the distribution of E-peak which is the energy at which the $v F_{v}$ spectrum reaches a maximum. To obtain the E-peak of a GRB, we fit its energy spectrum with a simple model (the so called Band's function) using the Xspec software.

In addition, this analysis provides the fluences of the GRBs detected within the field of view of FREGATE.

\section{THE E-PEAK DISTRIBUTION}

\section{Spectral fitting}

The Xspec software requires 3 input components: the observed spectrum is obtained by subtracting the background from the signal. It is coded on 128 channels in energy for the four detectors of FREGATE. The instrumental response is computed from a Monte Carlo simulation of the instrument, it has been extensively tested with ground calibrations and inflight calibrations with the Crab nebula [2]. The model spectrum is the model GRBM from Xspec, based on Band's function [3]:

$$
\begin{aligned}
& N(E)=A \cdot E^{\alpha} \cdot e^{-E / E_{o}} \quad \text { for } E<(\alpha-\beta) E_{o} \\
& N(E)=B \cdot E^{\beta} \quad \text { otherwise. }
\end{aligned}
$$

We can then calculate the E-peak energy $\left(E_{p}\right)$, the energy at which the $v F_{\mathrm{v}}$ spectrum has a maximum. $E_{p}$ is given by:

$$
E_{p}=E_{o}(2+\alpha)
$$

$E_{p}$ is defined when: $\alpha>-2$ and $\beta<-2$.

As an example the figure 1 shows the spectrum of GRB001225 which is well fitted by the Band's model. In comparison, the figure 2 shows a spectrum which can be fitted by a simple power law.

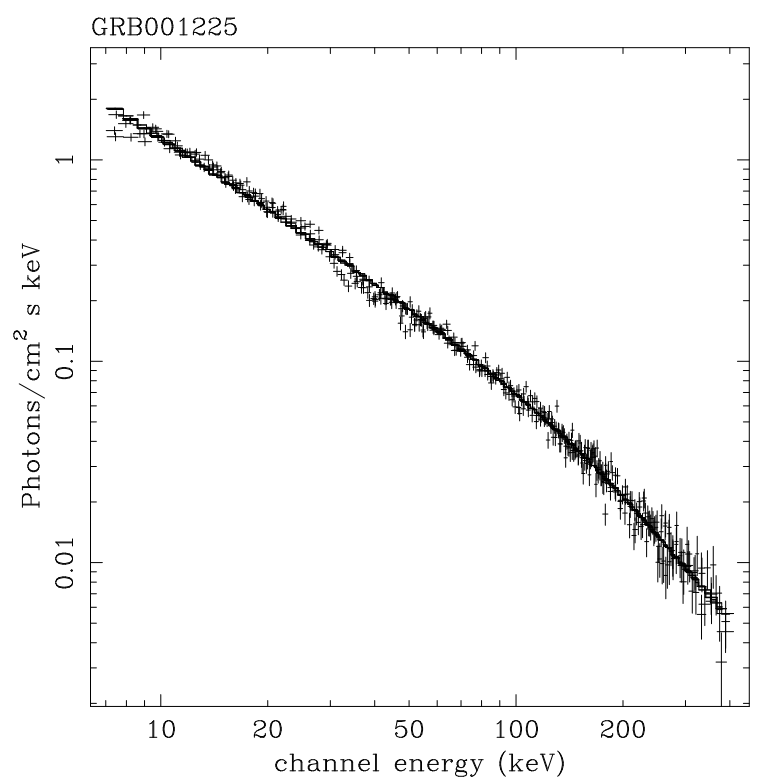

FIGURE 1. Spectrum of GRB001225 fitted with Band's function. $\alpha=-1.15, \beta=-1.90, E_{o}=277 \mathrm{keV}, E_{p}=235 \mathrm{keV}$ 


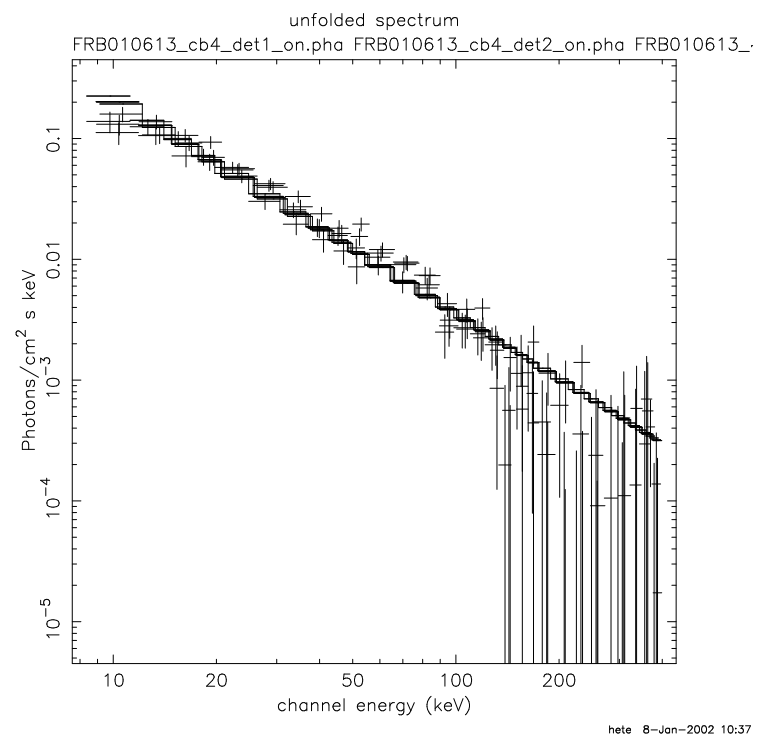

FIGURE 2. Spectrum of GRB010613 fitted with a power law. $\alpha=-1.78$

The table 1 lists the spectral parameters of 23 GRB candidates which occured within the FOV of FREGATE. Most of these events were confirmed by other GRB detectors on ULYSSES, KONUS, SAX-GRBM, RXTASM.

\section{The E-peak distribution}

The figure 3 shows the E-peak distributions mesured with the GRBs detected by FREGATE compared to BATSE and GINGA. The value of $E_{P}$ for FREGATE GRBs is obtained with Xspec, the distribution of BATSE comes from [4] and the GINGA's distribution was calculated using [5].

Some GRBs can be fitted by a simple power law (in that case $\beta$ is arbitrarely fixed at -10 ). When this is the case, if $\alpha \geq-2$, we consider that the data do not allow us to derive $E_{p}$ and we provide no value (see GRB010613). if $\alpha \leq-2$, the $v F_{V}$ spectrum is steadily decreasing in the energy range of FREGATE and we arbitrarily set $E_{p}$ to $10 \mathrm{keV}$.

The spectral fitting of FREGATE GRBs also allows us to compare the fluences in various energy ranges.

\section{The fluence}

The figure 4 shows the fluence in the range $30-400 \mathrm{keV}$ versus the fluence in the range $8-30 \mathrm{keV}$. The line $50 \%$ represente the place where there is as much fluence in the range $8-30 \mathrm{keV}$ as in the range $30-400 \mathrm{keV}$. These two

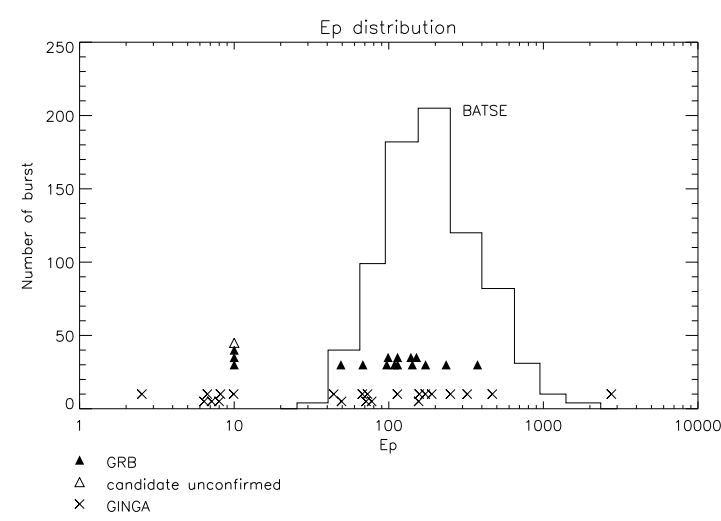

FIGURE 3. The E-peak distribution of the GRBs detected by FREGATE (triangles), GINGA (crosses) and BATSE.

fluences are well correlated for confirmed GRBs (with $80 \%$ of the fluence in the range $30-400 \mathrm{keV}$ on average).

A few events are very soft, having most of their fluence below $30 \mathrm{keV}$ (they are located below the line 50\%). The nature of these events is still unclear: genuine GRBs, very soft GRBs... see also [6]. According to a suggestion by J.Heise (GCN 1138), we call them X-Ray Flashes (XRFs).

For comparison, a few typical X-ray bursts have been added to this figure.

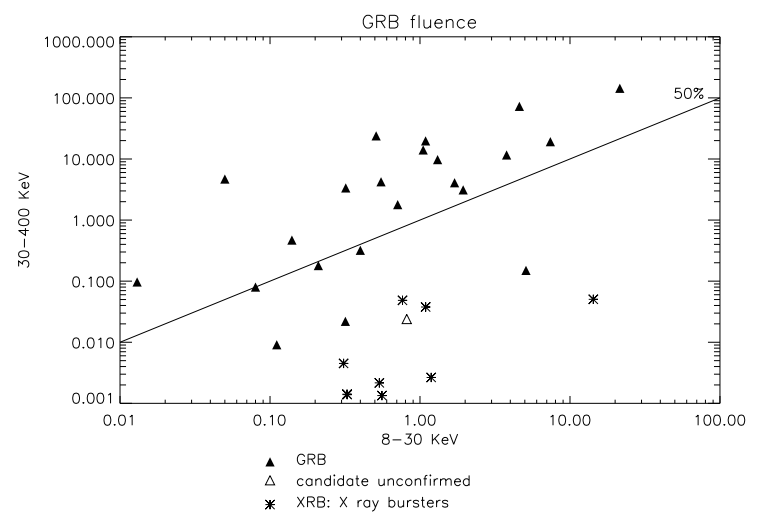

FIGURE 4. The fluence in $30-400 \mathrm{keV}$ vs the fluence in 8-30 $\mathrm{keV}$ for $23 \mathrm{GRBs}$ detected by FREGATE. The XRFs are placed under the line $50 \%$.

\section{CONCLUSION}

A preliminary study of 23 GRB candidates detected by FREGATE within its FOV shows the existence of a few soft events, XRFs (with $E_{p}<15 \mathrm{keV}$ and most of the fluence below $30 \mathrm{keV}$ ). These XRFs can be compared to 
TABLE 1. GRB candidates detected by FREGATE

\begin{tabular}{|c|c|c|c|c|c|c|c|}
\hline Sursauts & angle & $\begin{array}{c}\text { fluence } \\
8-400 \mathrm{keV}\end{array}$ & $\begin{array}{c}\text { fluence } \\
30-400 \mathrm{keV}\end{array}$ & $\alpha$ & $\beta$ & $\begin{array}{c}E_{O} \\
\mathrm{keV}\end{array}$ & $\begin{array}{c}E_{p} \\
\mathrm{keV}\end{array}$ \\
\hline GRB001102 * & $70^{\circ}$ & 77.33 & 72.33 & 0.14 & -2.86 & 58 & 142 \\
\hline GRB001225 & $37^{\circ}$ & 165.12 & 143.63 & -1.15 & -1.90 & 277 & 235 \\
\hline GRB010126 & $10^{\circ}$ & 2.50 & 1.79 & -.92 & -3.46 & 90 & 97 \\
\hline GRB010213 & $14^{\circ}$ & .12 & .0091 & -1.50 & -10 & 12 & \\
\hline GRB010225 & $23^{\circ}$ & .39 & .18 & -1.27 & -10 & 37 & \\
\hline GRB010326a & $60^{\circ}$ & 15.10 & 14.05 & -0.13 & -3.12 & 81 & 151 \\
\hline GRB010326b & $17^{\circ}$ & .16 & .08 & -2.13 & -10 & 50 & 10 \\
\hline GRB010612 & $13^{o}$ & 4.78 & 4.23 & -0.90 & -10 & 189 & \\
\hline GRB010613 & $38^{\circ}$ & 26.58 & 19.18 & -1.15 & -10 & 80 & \\
\hline GRB010629 & $28^{\circ}$ & 5.06 & 3.12 & -1.41 & -3.50 & 83 & 49 \\
\hline GRB010921 & $44^{o}$ & 15.41 & 11.63 & -1.42 & -2.35 & 170 & 99 \\
\hline GRB010923 & $60^{\circ}$ & 5.78 & 4.08 & -0.57 & -3.63 & 80 & 114 \\
\hline GRB010928 & $24^{\circ}$ & 20.86 & 19.77 & -0.64 & -1.82 & 276 & 375 \\
\hline GRB001115a & $30^{\circ}-150^{\circ}$ & 24.41 & 23.90 & 0.53 & -1.55 & 55 & 139 \\
\hline GRB001115b & & 11.07 & 9.76 & -0.91 & -1.42 & 105 & 114 \\
\hline GRB010428 & $4^{o}-82^{o}$ & .34 & .022 & -2.06 & -10 & 12 & 10 \\
\hline GRB010609 & $48^{\circ}-195^{\circ}$ & 5.24 & .15 & -3.14 & -10 & 11 & 10 \\
\hline $\mathrm{GRB}^{2} 10621^{\dagger}$ & & .84 & .024 & -2.31 & -10 & 10 & 10 \\
\hline GRB010706 & & .11 & .097 & 0.29 & -10 & 58 & \\
\hline GRB010827a & & .61 & .47 & -.79 & -2.44 & 56 & 68 \\
\hline GRB010828 & $46^{o}-113^{o}$ & .72 & .32 & -0.96 & -10 & 176 & \\
\hline GRB010903 & $53^{o}-106^{o}$ & 3.68 & 3.36 & 0.10 & -3.53 & 52 & 109 \\
\hline GRB010917 & & 4.74 & 4.69 & 0.99 & -1.69 & 58 & 173 \\
\hline
\end{tabular}

* This burst was in the limit of the field of view of FREGATE

${ }^{\dagger}$ this events have been detected only by FREGATE

the detection of such soft events by GINGA and the WFC on Beppo SAX.

In its second year of observations, HETE-2 will provide fast localizations of several new XRFs, an approach which may shed a new light on the nature of these enigmatic sources. characteristics of GINGA gamma ray bursts, Ap.J, 500:873-887 (1998).

6. J.Heise, J. in't Zand, R. M. Kippen and P. M. Woods. $X$-ray flashes and $X$-ray rich Gamma Ray Bursts, Astro$\mathrm{ph} / 0111246$ (2001).

\section{REFERENCES}

1. Atteia \& al, these proceedings.

2. Olive $\&$ al, these proceedings.

3. Band \& Al, Ap.J, 413:281-292 (1993).

4. J. J. Brainerd, G. Pendleton, R. Mallozzi, M. S. Briggs, R. D. Preece. The BATSE Gamma-Ray Burst E-Peak Distribution: unpublished manuscript available by request from Jim Brainerd at the e-mail address <brainjj@bellsouth.net>.

5. T. E. Strohmayer \& E. E. Fenimore. X-ray spectral 